\title{
Parameterized Comparative Analysis of Various Lossy and Lossless Image Compression Practices
}

\author{
Anshuma Patel ${ }^{1}$, Sanjiv Kumar Shriwastava ${ }^{2}$ \\ Research Scholar, ECE Department, SBITM, Betul, India ${ }^{1}$ \\ Principal, SBITM, Betul, India ${ }^{2}$
}

\begin{abstract}
In this digital globe, the need of successful generation and transmission of data in numeric form is highly desirable. Images have always gathered attention as the data can be represented in a more appreciable format as compared to long strings of characters, so is the necessity of excellent image compression. The theory below demonstrates various image compression techniques available and briefly compares them all on the basis of certain parameters like PSNR, Compression Ratio, MSE and Code Energy. We have also proposed an innovative development in the arena of lossy image compression called as Lattice Vector Quantization which is supposed to reduce the average computational time required for image compression as well as the memory requirement for the same.
\end{abstract}

Keywords: LVQ, PSNR, MSE, Compression ratio.

\section{INTRODUCTION}

We live in the digital era where there is incontestable need of representing the generated data and transmitting them reliably. Source encoding is the process by which data representation is done, source encoder performs the task and reliable transmission is achieved with the help of channel encoder and decoder. Thus source coding and channel coding both are very imperative process of digital communication systems. In this work Lattice Vector Quantization is used for source coding as well as for channel coding, taking image as source. Images are one of the important means of conveying or transmitting information. They pithily convey information about sizes, position and inter-relationships between objects. An image is worth a thousand words.

\section{Image Compression Practices}

Image compression is a process of attaining a squashed representation for the image without any reduction in the perceptual quality by reducing the number of bits used to represent an image.

Image compression can be done in two ways i.e. lossy image compression and lossless image compression. Lossless compression allows the actual data to be absolutely reconstructed from compressed data and ideal in the field of medical imaging, technical drawing etc. By contrast lossy compression is the method suitable for natural images where slight loss of reliability is acceptable in order to achieve significant drop in the bit rate.

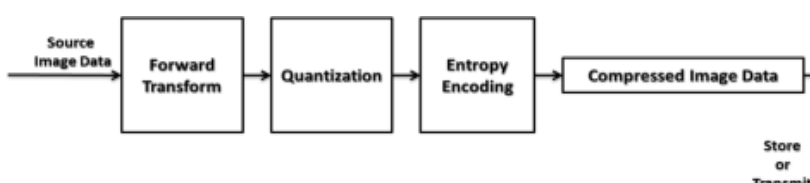

(a)

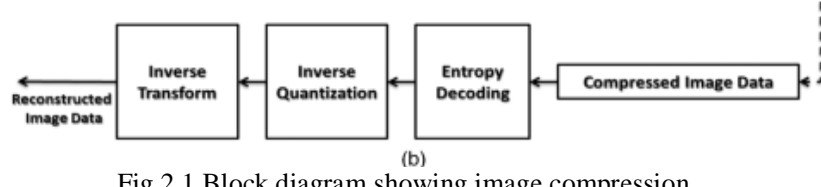

Fig.2.1 Block diagram showing image compression
A. Lossless Compression:

Lossless compression techniques are:

1. Run-length encoding: in this process the series which contain the same valued data in many successive data elements are stored as a sole data value instead of the original series.

For example, for a sequence of data having frequent runs of zeros, run length encoding can be done as.

Original data stream:

$\begin{array}{llllllllllllllllllll}12 & 4 & 84 & 2 & 0 & 0 & 0 & 0 & 56 & 2 & 0 & 0 & 1 & 23 & 45 & 7 & 0 & 26 & 46 & 7\end{array}$

$\begin{array}{llllll}9 & 0 & 0 & 0 & 22 \ldots \ldots . .\end{array}$

Run length encoded:

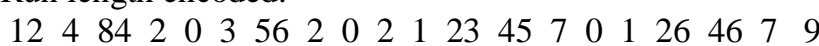
$0322 \ldots$

Here every time when a zero come across in the input stream, describe with the help of two values one is a zero (act as a flag which shows the beginning of RLE) and the other value tell the number of consecutive zeros. The main problem with this method is that for compression to take place the average run length must be longer than two, if not then the single zeros will make the original file larger.

2. DPCM based compression: DPCM is a signal encoder which uses the basics of PCM i.e. Pulse Code Modulation represents the analog signals digitally. Here it sample amplitude of analog signal regularly at a uniform intervals and is then quantized to the nearby value within the digital steps range. DPCM add some more features in PCM to compress the data to certain instinct i.e.it does not encode the original stream but the difference between the current and the predicted value, thus also known as predictive coding and the prediction is based on the preceding value, and this difference gets only stored in the encoder .thus less number of bits can be used to represent the same information. This above procedure is described using the figure below Fig.2.2. 


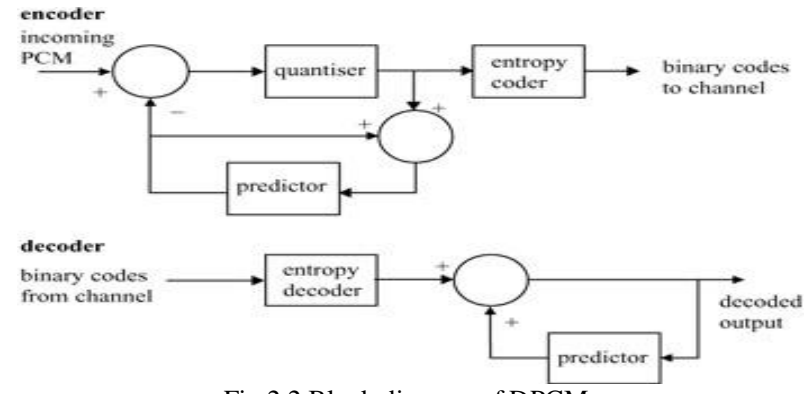

Fig.2.2 Block diagram of DPCM

3. Deflation based compression: it is the combination of LZ77 and Huffman coding technique. The deflate sequence consist of series of block which are preceded by three bit header

\section{First bit:}

1: last block in the stream

0: more block to process.

\section{Second and third bit:}

00: stored section of length between 0 to 65,535 bytes

01: static compressed block (Huffman compressed), using pre-agreed Huffman tree

01: compressed block complete using Huffman table

11:don't use, reserved.

Compression include following two steps.

Duplicate string elimination: This is the first step of Deflation Based Image Compression. During this process a back reference is inserted whenever a duplicate series of bytes exists within compressed block. This back reference links the identical string's previous location. This procedure is accomplished using LZ77 [1] and LZ78 [2] methods.

Bit Reduction: This is the second step of Deflation Based Image Compression Technique. The Huffman Coding [3] is used to imply this method. It performs the process of replacing the frequently used symbols with the shorter representation i.e. less number of bits and less common used symbols with longer representation i.e. comparatively more number of bits. Huffman coding is the method used to generate an un-prefixed tree of non overlapping intervals in which the probability of the symbol needing to be encoded is inversely proportional to the sequence length.

4. Entropy encoding based compression: The most commonly used entropy encoding techniques are Huffman and arithmetic coding which basically generates and assigns a unique prefix free code to each unique symbol that arise in input stream. Prefix free code is not error correcting code but is variable length uniquely decodable code which are differ by other codes in the sense that it possess the property of prefix i.e. requirement that no code word is prefix of any other code in the system.

An illustration: a code define by the code word $\{8,77\}$ which has prefix property and a code with the code word $\{8,7,78,77\}$ does not exhibit the prefix property since 7 is not the prefix of 78 and also of 77 .These prefix codes are used to transmit the message as a sequence of concatenated code word without any requirement of special markers in between the words to frame them in message, then entropy encoder compresses the data with the replacement of each fixed length symbol by the corresponding variable length prefix free code word. After generating and assigning prefix code i.e. after compressing the message with the help of prefix code, it is then again encoded by channel coding scheme before transmission.

5. Chain codes based compression: Compression based on chain codes including the encoding of the connected component in the image separately i. e. connected component or 'blob' can be defined as, within the non empty topological space the maximal connected subsets. For that a particular point is selected on the boundary whose coordinates are transmitted, encoder at each step moves along the boundary of the blob and transmits the symbol representing the direction in which movement takes place. The process continues until it returns to the starting point at which the blob has been completely covered after that next blob gets encoded following the same procedure. It can be a efficient method for image has less number of large connected components.

\section{B. Lossy Compression:}

1. Fractal based compression: In 1987 Michael Barnslay introduced the term fractal compression. Later in 1992 Barnsley and Alan Sloan implemented the first practical Fractal compression algorithm. It's a fractal based compression method. Fractal can be a mathematical set or can be considered as a natural phenomenon which exhibit an identical pattern at uniform interval. If this repeated pattern is exactly same at every scale then it is considered as a self similar pattern. Sometime they possess the property of being same at different interval. This technique is particularly suitable for such images that possess certain sections which often resembles other parts of same image.

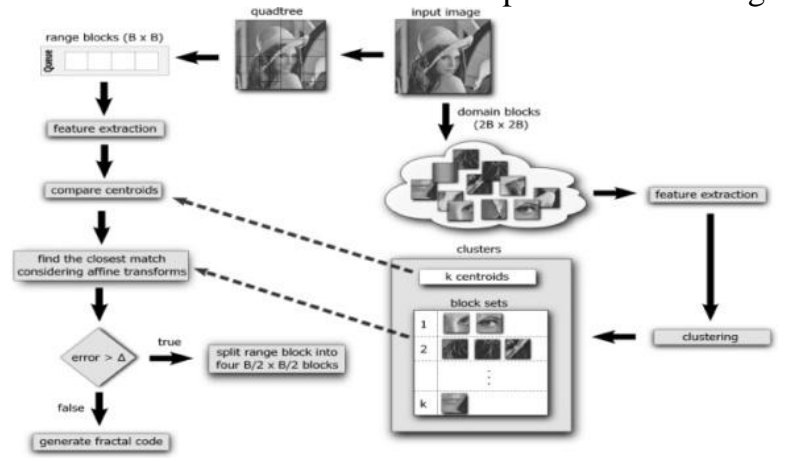

Fig.2.3 Block diagram showing the procedure followed in Fractal Compression

This algorithm translates replicated parts into mathematical information known as fractal codes and is used to reconstruct the already encoded images. Decoding is quite fast but having a disadvantage of being computationally expensive as finding the self similarities in an image is a quite complicated process, which makes it unfeasible. This algorithm provides a unique property to image after converting it to fractal code i.e. the property of 
being independent and this property is used to enhance the 4. Transform based compression: Fourier based display resolution of target image and is termed as Fractal transform like DCT (Discrete Cosine transform) is most interpolation.

2. Chroma Sub sampling: Chroma sub sampling based compression takes the advantage of human visual's system lower sensitivity for color than for luminance[4]. It compresses the image by implementing less bandwidth to chroma function than to luma function. An image consist of two parts chroma and luma.

$$
\begin{aligned}
& \text { Luminance Chrominance } \\
& \text { Luma = Brightness value } \\
& \text { Chroma = Color value }
\end{aligned}
$$

Chroma is the word derived from chrominance which covey the color information of picture, represented by two color difference component $\mathrm{U}=\mathrm{B}^{\prime}-\mathrm{Y}^{\prime}$ (blue - luma) and $\mathrm{V}=\mathrm{R}^{\prime}-\mathrm{Y}^{\prime}$ (red - luma). It is different from luma which defines the brightness value of picture.

Sampling is done using $\mathrm{j}: \mathrm{a}: \mathrm{b}$ where the first number, is the value for the luminance (i.e. brightness whose value is "4" in most cases although some formats have a " 3 ") sampling rate, and the next terms in the nomenclature have to do with color sampling of blue and red aspects correspondingly.

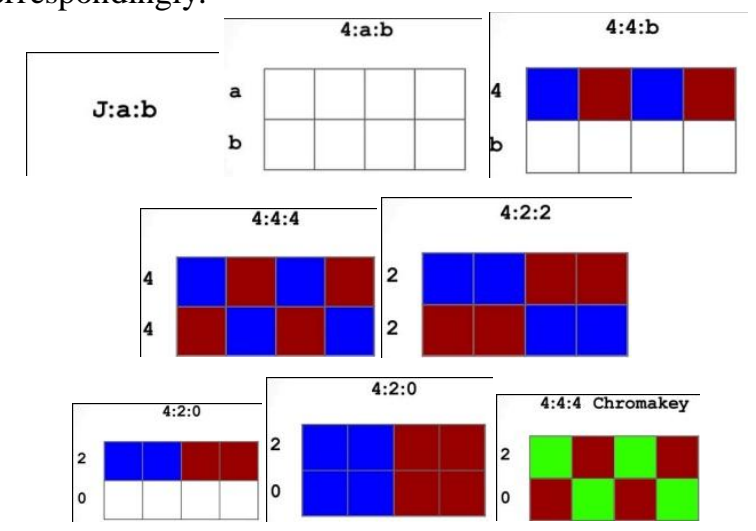

This sub sampling reduce the size of image by $50 \%$ with the minimal loss of quality but at the cost of removal of redundant chroma information and the final size of compressed image is considerably low.

3. Color space based compression: Color based compression follow the process of reducing the color space to most common colors in image. Specific organization of colors is color space which allows the reproducible representation of color. Real time images are usually stored in RGB color space [5], but transmitting them in RGB color space format require high bandwidth requirement which is impractical and the solution to this problem is to convert RGB color space into other color space like YUV,YIQ and YCbCr. As RGB information generated by devices like scanners, cameras etc all color space can be derived from them.[5].This color based compression can be combine with dithering i.e. the process of intentionally introducing noise which randomize quantization error in order to avoid posterization with abrupt changes from one tone to another which entails the conversion of continuous gradation of tone to several regions of fewer tones. transform like DCT (Discrete Cosine transform) is most
commonly method for image compression [6], wavelet transform is also which is followed by quantization and entropy encoding.

i.DCT based: it follows the procedure of separating the image into different frequencies during the step called quantization where it discard the less important frequencies and only important frequency is used to reconstruct the original image.

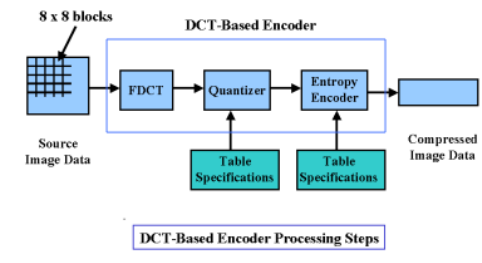

ii. Wavelet based: wavelet exhibit a special property due to which it allow simultaneous time and frequency analysis like wave [7]. Wavelet is a small wave that has energy concentrated in time. For compression firstly it digitizes the image and converts it into string of numbers, after that it decomposes the image into sequence of wavelet coefficient. Then this wavelet coefficient is converted into another wavelet coefficient using the principle of thresholding i.e. the procedure in which coefficients that are close to or equal to zero are modified into that which contains long string of zeros. And finally entropy encoding is applied.

\section{III.QUANTIZATION}

Quantization can be defined as the process of converting the continuous set of information in the image data with a finite set. It has an important role in distortion of reconstructed image. It is an irreversible process and is a source of information loss. Quantization may be classified as:

1. Scalar Quantization

2. Vector Quantization

1. Image compression using scalar quantization

Scalar Quantization, denoted by $y=Q(x)$, is the process of representing a very large or possibly infinite, set of values with a much smaller finite set of value. Shapiro was the introducer of such a technique with is Embedded Zerotree Wavelet (EZW) [8] algorithm. Many improved algorithms are also proposed in this field. Said \& Pearlman [9] improved the EZW algorithm by presenting by different implementation based on a set partitioning sorting algorithm called the SPIHT [9], which provided an even better performance than the improved version of EZW. .

Scalar quantized methods employ some sets or groups of pixels, in which the set is tested to determine whether the maximum magnitude in it is above a certain threshold. The results of these significance tests determine the path taken by the coder to code the samples. Some principles used are the partial ordering of magnitude coefficients with a set-partitioning sorting algorithm, exploitation of self-similarity across different scales of an image wavelet 
transform and bit plane transmission in decreasing bit plane order.

The image coding scheme the SPECK [10], in that it makes use of sets in the form of blocks, it does not exploit the similarity and use the tree span, across different sub bands; rather, It has its roots primarily in the ideas developed in the SPIHT, and few block coding image coding algorithms. Merits and demerits of these methods [10] are stated as follows:-

a. $\quad$ Embedded Zerotree Wavelet (EZW) :-

Features: •Employs progressive \& embedded transmission

- Uses zerotree concept

- Tree coded with single symbol

- Uses predefined scanning order

-Good results without prestored tables, codebooks, training

Demerits: - Transmission of coefficient position is missing

- No real compression

- Followed by arithmetic encoder

b. Set Partitioning in Hierarchical Trees (SPIHT)

:- Features: •Widely used high PSNR values for given

Compression Ratios for variety of images

- Hierarchical trees are set - partitioned

- Employs spatial orientation tree structure

- Keeps track of states of sets of indices using 3 lists: LSP, LIS, LIP

- Progressive and embedded transmission is used.

- In perceptual image quality and PSNR, superior to JPEG

Demerits: • Only implicitly locates position of significant coefficients

- Due to 3 lists more memory requirements

- Single bit is used to form transmitted

information.

- Suits variety of natural images

- Perceptual quality not optimal

c. $\quad$ Set Partitioning Embedded bloCK (SPECK) :Features: - Does not use trees

- Uses blocks- rectangular regions

- Exploits clustering of energy in frequency and space

-Progressive and embedded transmission is used.

- Low computational complexity

- Employ quad tree and octave band partitioning

- Low memory requirements due to 2 lists

- Better PSNR than SPIHT

3. Image compression using Vector Quantization: In the process of quantization, the entire vector gets replaced with the vectors chosen from sparse non uniform set. Now this vector stores only its indices into sets. Hence provides much more compact representation of the image. This is nothing but the set of possible quantized values for the vectors are called a "codebook".

Vector quantization (VQ) is a lossy data compression method based on the principle of block coding. A VQ is simply an approximation. The idea is similar to that of "rounding-off" (say to the nearest integer).

Copyright to IJARCCE
Vector quantization allows the modeling of probability density functions by the distribution of archetype vectors. It works by dividing a large set of points (vectors) into groups represented by centroidpoint having approximately the same number of points closest to them. . It is used for lossy data correction, lossy data compression, and density estimation.

Vector quantization, also called "block quantization" or "pattern matching quantization" is often used in lossy data compression. It works by encoding values from a multidimensional vector space into a finite set of values from a discrete subspace of lower dimension. A lowerspace vector requires less storage space, so the data is compressed. Due to the density matching property of vector quantization, density is the function that is inversely proportional to the compressed data error. Vector Quantization (VQ) has became the powerful tool and is best explained with a numerical example by Y. Linde, A. Buzo \& R. M. Gray in 1980 [11].

\section{Disadvantages of VQ}

1. The encoding process is computationally intensive and slow, which increases exponentially with the vector dimension.

2. With increasing vector dimension and codebook size, memory storage requirement for the codebook also increases rapidly.

3. With large vector dimensions and codebooks, codebook generation is a very lengthy process.

TABLE I

COMPARISON OF DIFFERENT IMAGE COMPRESSION TECHNIQUE

\begin{tabular}{|c|c|c|c|c|c|}
\hline $\begin{array}{l}\text { S. } \\
\text { n } \\
\text { o }\end{array}$ & $\begin{array}{l}\text { Compression } \\
\text { method }\end{array}$ & Psnr & $\begin{array}{l}\text { Mean } \\
\text { square } \\
\text { error }\end{array}$ & $\begin{array}{l}\text { Energy of } \\
\text { code }\end{array}$ & $\begin{array}{l}\text { Compression } \\
\text { ratio }\end{array}$ \\
\hline \multicolumn{6}{|c|}{ Lossless } \\
\hline 1 & $\begin{array}{l}\text { Runlength } \\
\text { encoding }\end{array}$ & High & low & Less & low \\
\hline 2 & DPCM based & $\begin{array}{l}\text { low psnr can } \\
\text { be achieved }\end{array}$ & high & $\begin{array}{l}\text { can be } \\
\text { decreased }\end{array}$ & $\begin{array}{l}\text { high cr can be } \\
\text { achived }\end{array}$ \\
\hline 3 & $\begin{array}{l}\text { Deflation } \\
\text { based }\end{array}$ & is not too low & $\begin{array}{l}\text { is not too } \\
\text { high }\end{array}$ & $\begin{array}{l}\text { is not too } \\
\text { high }\end{array}$ & is not too high \\
\hline 4 & $\begin{array}{l}\text { Entropy } \\
\text { encoding }\end{array}$ & $\begin{array}{l}\text { huffman-low } \\
\text { arithematic- } \\
\text { high }\end{array}$ & $\begin{array}{l}\text { huffman- } \\
\text { high } \\
\text { arithmatic- } \\
\text { low }\end{array}$ & $\begin{array}{l}\text { huffman- } \\
\text { high } \\
\text { arithmatic } \\
\text {-low }\end{array}$ & $\begin{array}{l}\text { huffman-high } \\
\text { arithmatic-low }\end{array}$ \\
\hline 5 & $\begin{array}{l}\text { Chain code } \\
\text { based }\end{array}$ & Low & high & high & high \\
\hline \multicolumn{6}{|c|}{ Lossy } \\
\hline 1 & Fractal based & 27.21 & $\gg>27.21$ & $\gg>32$ & $>>32$ \\
\hline 2 & $\begin{array}{l}\text { Chroma } \\
\text { subsampling }\end{array}$ & High & low & Low & low \\
\hline 3 & $\begin{array}{ll}\begin{array}{l}\text { Color } \\
\text { based }\end{array} & \text { space } \\
\end{array}$ & 54.86 & >>54.86 & high & high \\
\hline 4 & $\begin{array}{l}\text { Transform } \\
\text { coding }\end{array}$ & 36.71 & $\gg>36.13$ & $>=16$ & $>=16$ \\
\hline 5 & $\begin{array}{l}\text { Vector } \\
\text { Quantization }\end{array}$ & 28.26 & $\gg 28.26$ & $<32$ & $<32$ \\
\hline
\end{tabular}

\section{IV.LATTICE VeCTOR QUANTIZATION}

The Lattice Vector Quantization is a development over conventional Vector Quantization technique for compression of lossy images. In this technique, the lattice based vector quantizer encodes the source vectors by mapping them into the lattice points. Since lattice is a regular structure hence, there is no need to generate and store any kind of codebook. It makes the encoding and decoding very simple as selection of the nearest codeword for a given input becomes very straightforward. This is an extension of the uniform scalar quantization, offers some 
advantages over the classical vector quantization. It reduces the computational time for comparable performances and no memory is required to store the codebook.

Lattice Vector Quantization performance depends on the length of the codebook. If the number of lattice points is less in a codebook, the time required to get quantized lattice point will be less but at the same time quality of reconstructed image will be degraded. If the size of the codebook is larger then the time required to search nearest vector will be more. So to avoid these situations, one solution is expansion of codebook when needed. This technique eliminates overload error in the codebook. In comparison with single codebook system, a pyramidal codebook structure can also be used, which in turn will require less time for encoding and decoding.

\section{Conclusion}

In this paper, a comparative analysis of various image compression techniques has been done. This comparison is based on four elementary parameters of image quality assessment i.e. PSNR, Compression Ratio, Code Energy and Mean Square Error. All the results have been tabulated from which the pros and cons of image compression using various algorithms can be inferred.

For lossy image compression, a new technique of Lattice Vector Quantization is proposed, which is capable of conquering the disadvantages of traditional Vector Quantization technique. This technique is supposed to reduce the computational time as well as the memory requirement for the picture compression.

\section{REFERENCES}

[1] Ziv, Jacob; Lempel, Abraham (May 1977). "A Universal Algorithm for Sequential Data Compression". IEEE Transactions on Information Theory 23 (3): 337-343.

[2] Ziv, Jacob; Lempel, Abraham (September 1978). "Compression of Individual Sequences via Variable-Rate Coding". IEEE Transactions on Information Theory 24 (5): 530-536.

[3] Huffman, D. (1952). "A Method for the Construction of MinimumRedundancy Codes". Proceedings of the IRE 40 (9): 1098-1101.

[4] S. Winkler, C. J. van den Branden Lambrecht, and M. Kunt (2001). "Vision and Video: Models and Applications". In Christian J. van den Branden Lambrecht. Vision models and applications to image and video processing. Springer. p. 209. ISBN 978-0-7923-7422-0.

[5] E. Prathibha, Dr.A.Manjunath, Likitha.R , RGB to YCbCr Color Conversion using VHDL approach, International Journal of Engineering Research and Development ISSN: 2278-067X, Volume 1, Issue 3 (June 2012), PP.15-22.

[6] M. Sima, S. Vassiliadis, S. Cotofana and J. T.J. Van Eijndhoven, “ Color space conversion for MPEG decoding on FPGAaugmented trimedia processor", Proceedings IEEE International Conference on Application-Specific Systems, Architectures and Processors, pp. 250-259,June 2003.

[7] N. Ahmed, T. Natarajan and K.R.Rao, "Discrete Cosine Transform," IEEE Trans. Computers, 90-93, Jan. 1974.

[8] Jerome M. Shapiro, "Embedded Image Coding Using Zerotrees of Wavelet Coefficients," IEEE Transactions on Signal Processing, Vol. 41, No. 12, December 1993.

[9] Amir Said and William A. Pearlman, "A New, Fast and Efficient Image Codec Based on Set Partitioning in Hierarchical Trees," IEEE Transactions on Circuits and Systems for Video Technology, Vol. 6, No. 3, June 1996.

[10] William A. Pearlman, Asad Islam, Nithin Nagaraj, and Amir Said, “ Efficient, Low-Complexity Image Coding with a Set-Partitioning Embedded Block Coder," IEEE transaction Circuits and Systems for Video Technology, Vol.14, No.11, pp. 1219-1235, 2004.

[11] P. C. Cosman, R. M. Gray, M. Vetterli, "Vector Quantization of Image Subbands: A Survey", IEEE transaction on Image Processing. 